\title{
Hybrid Method for the Formation of Biocomposites on the Surface of Stainless Steel Implants
}

\author{
Sergei I. Tverdokhlebov, Viktor P. Ignatov, Igor B. Stepanov, Denis O. Sivin, Danila G. Petlin \\ Tomsk Polytechnic University, Tomsk, Russia \\ Email: tverd@tpu.ru
}

Received July 29, 2012; revised August 30, 2012; accepted September 10, 2012

\begin{abstract}
This study reports a hybrid method which allows the formation of biocomposites on stainless steel implants. The main idea of the method is to create multilayer coatings consisting of titanium primer layer and a microarc calcium-phosphate coating. The titanium layer is deposited from plasma of continuous vacuum-arc discharge, and calcium-phosphate coating is formed by the microarc oxidation technique. The purpose of the hybrid method is to combine the properties of good strength stainless steel with high bioactivity of calcium-phosphate coating. This paper describes the chemical composition, morphology characteristics, adhesion and the ability of the formed biocomposites to stimulate the processes of osteoinduction. It is expedient to use such biocomposites for implants which carry heavy loads and are intended for long-term use, e.g. total knee endoprosthesis.
\end{abstract}

Keywords: Biocomposite; Stainless Steel; Titanium; Vacuum-Arc Deposition of Coatings; Short-Pulse High-Frequency Plasma-Immersion Ion Implantation; Microarc Oxidation; Implant

\section{Introduction}

One of the goals of the field of medical materials is the creation of new structural materials which can provide optimal biomechanical properties of implants widely used in modern traumatology, orthopedics and dentistry.

Most modern implants are made of metals, because they have good biomechanical characteristics and provide a sufficiently reliable fixation of the injured bones. The advantages of this approach are connected with the high level of elaboration of the manufacturing technology. However, the usage of such medical devices does not provide a fundamental improvement according to the results of musculoskeletal system injuries treatment. To increase the speed of recovery it is necessary to influence the process of reparative osteogenesis directly using the osteoplastic materials such as calcium phosphates.

However, the mechanical properties of calcium-phosphates materials such as high fragility and low wear resistance do not allow using them as biomaterials that carry serious loads. The low mechanical properties are the distinctive feature of highly porous $\mathrm{CaP}$ ceramics. But the presence of pores larger than 80 microns is considered as a necessary requirement for sprouting of blood vessels and bone tissue cells [1]. Moreover, the mechanical strength of the implant and bone ingrowth should remain constant during the whole regeneration process.

It becomes clear that single-phase biocompatible ma- terials cannot provide all the necessary functions of bones or other calcified tissues, and therefore there is a need to create multiphase biomaterials with the structure and composition acting like a natural bone. Such materials are classified as hybrid, which can be formed by combination of chemically different components. To merge all the positive properties of the hybrid system components it is necessary to integrate their parts in one material. Hybrid materials include composite materials, multilayer systems, particles and fibers with modified surface [2].

The best way for implants carrying heavy mechanical loads is the creation of composite materials in which the mechanical strength is provided by metallic base and $\mathrm{CaP}$ coatings provide bioactive properties, positively influence on the osteogenesis process and reinforce the fixation of the implant in bone tissue [3].

There are several different methods to form $\mathrm{CaP}$ coatings such as plasma spraying [4], electrophoresis [5], solgel method [6], biomimetic method [7], microarc oxidation $[8,9]$, etc.

The simplest, cost effective, well-studied and widely used way to form $\mathrm{CaP}$ coatings on titanium implants is the electrochemical method [9]. The method of microarc oxidation (MAO) allows the formation of $\mathrm{CaP}$ coating with sufficient thickness and high adhesion with the metallic base. High level of biocompatibility of MAO $\mathrm{CaP}$ coatings on $\mathrm{Ti}$ is shown in different articles $[9,10]$. 
However, this method has a limitation connected with the fact that MAO coating can be formed only on the valve group materials ( $\mathrm{Ti}, \mathrm{Zr}, \mathrm{Nb}$, etc.). As for the endoprostheses of big joints, it is preferable to use stainless steel and its alloys as a metallic skeleton, because $\mathrm{Ti}, \mathrm{Nb}$, $\mathrm{Zr}$ are inferior to them in strength characteristics.

The most prevalent methods of forming $\mathrm{Ti}$ coatings are plasma spraying and condensation of materials from the plasma of vacuum-arc and magnetron discharges $[11,12]$. Titanium layer allows carrying out the MAO process in electrolyte containing calcium and phosphorus compounds. The main feature of the MAO process is the mixing of oxidizing material with ions of calcium and phosphorus coming from the electrolyte. As a result, the formation of $\mathrm{CaP}$ composite on the basic material occurs. A method that implies the formation of the valve group material coating of certain thickness and then its microarc oxidation was proposed in Tomsk Polytechnic University (TPU) and was patented in Russian Federation [13]. In order to reach high adhesion of the titanium layer with the basis, it was proposed to sputter the titanium with vacuum techniques, e.g. vacuum-arc evaporator.

In preliminary experiments the $\mathrm{Ti}$ layer was deposited on a steel substrate by magnetron sputtering. It was found out that the Ti layer with thickness of about $1.5 \mu \mathrm{m}$, formed from plasma of pulsed magnetron discharge, does not provide the conditions for the following microarc oxidation process. With such thickness the depth of the MAO breakdown discharge reached the steel substrate with the formation of iron oxides, which are extremely unstable in the oxidizing environment.

To form the $\mathrm{Ti}$ layer on the substrate the Complex Plant for the implementation of hybrid technologies of ion-beam and ion-plasma modification of materials was used [14], which provided the obtaining of the sufficient thickness of $\mathrm{Ti}$ layer for the MAO process, good adhesion and technologically justified rate of its deposition.

\section{Materials and Methods}

Samples prepared for research were made of stainless steel $(12 \mathrm{X} 18 \mathrm{H} 9 \mathrm{~T})$ of the following size: $15 \times 15 \times 2 \mathrm{~mm}$.

Intermediate titanium coating from plasma of continuous vacuum-arc discharge in terms of short-pulse, highfrequency plasma-immersion ion assisting was formed on the Complex Plant (CP) in TPU [14].

Technological capabilities of $\mathrm{CP}$ allow organizing a new level of implementing the wide range of hybrid technologies of ion-beam and plasma processing of materials, including cleaning and activation of the surfaces, highintensity and high concentration ion implantation, the formation of wide transition layers between the substrate and the coating, the deposition of coatings in conditions of intense ion mixing on the conducting and insulating materials.

During the formation of the primer valve group metal on the surface of stainless steel CP was equipped with four vacuum arc plasma generators with titanium cathodes, the gas plasma generator and short-pulse highfrequency bias voltage generator.

The preparation of the samples before the formation of the titanium layer included mechanical grinding, polishing with abrasive pastes, chemical cleaning in the ultrasonic bath with a solution of carbon tetrachloride and subsequent drying.

The final preparation of samples for the coating formation was carried out in vacuum on $\mathrm{CP}$. To provide the uniform sputtering of the coating on the surface of stainless steel samples were placed in holders, clamped on the planetary transferring mechanism. Samples rotational speed was $10 \mathrm{rev} / \mathrm{min}$.

Regimes of ion cleaning, heating and surface activetion have been implemented using an Ar plasma, formed by a gas plasma generator with a hot cathode, gas flow rate was $1.5 \mathrm{l} / \mathrm{h}$. Current of vacuum arc discharge was equal $(20$ - 30) A. Ion etching of the stainless steel surface was carried out with high frequency $(100 \mathrm{kHz})$ formation of bias potential with $(1-4) \mathrm{kV}$ amplitude on the surface of the sample, pulse duration was $7 \mathrm{~ms}$ and their filling factor was $70 \%$. The pressure of the reaction gas in the vacuum volume varied from 0.8 to $1.5 \mathrm{~Pa}$. The sample temperature was maintained at $450^{\circ} \mathrm{C}$.

At the final stage of preparation of the surface the metal plasma generators had the discharge current equal to $200 \mathrm{~A}$. The stage of the formation of the transition layer between the base material and Ti coating was carried out in the dynamic change of the pulse bias potential from -2.5 to $-0.5 \mathrm{kV}$.

The formation of Ti coating was carried out in plasma of continuous vacuum-arc discharge, pressure was equal to $10^{-3} \mathrm{~Pa}$, and the temperature in vacuum chamber was $350^{\circ} \mathrm{C}-400^{\circ} \mathrm{C}$. Cathode was made of Ti VT1-0. The samples were under a constant negative bias voltage which was ranged from 90 to $500 \mathrm{~V}$. The ion current density was $30 \mathrm{~mA} / \mathrm{cm}^{2}$. The rate of formation of coatings was $0.33 \mu \mathrm{m} / \mathrm{min}$; time-up to 120 minutes.

Bioactive $\mathrm{CaP}$ coating was formed by MAO under pulsed or direct current in the conditions of spark or arc discharges in a solution of phosphoric acid (concentration is $5 \%-33 \%$ ) with the addition of calcium compounds in the oversaturated state. Due to the mixing of the base material with titanium, and the latter with the CaP coating, the biocomposite structure was created.

The MAO process is the oxidation of intermediate titanium layer with oxygen, which is produced during electrolysis, and the interaction of the formed oxide layer with the elements of the electrolyte in plasma of microarc discharges. As a result, the formation of the calcium- 
phosphate composite on the implant's material occurs.

Qualitative and quantitative phase analysis of the formed biocomposites was carried out using X-ray DRON-3M diffractometer, EVA search program with PDF-2 database of powder samples. The investigation of the biocomposite's morphology and elemental analysis were performed on Philips SEM 515 scanning electron microscope with an integrated system for energy dispersive analysis. Energy dispersive analysis of the transverse section of the biocomposite coating was carried out on samples obtained by the fracture technique.

The thickness of the coatings was determined by the "spherical microsection" method on "CSM Calotest" equipment. Measurement of hardness and elastic modulus of the surface layer was carried out using "CSM NanoHardness Tester" measuring device.

The analysis of the adhesion strength of the Ti coatings was performed by the scratch-test method using "Micro Scratch Tester MST-S-AX-0000" device.

The morphology study of the coatings and surface roughness measurements were made using "MICROMEASURE 3D Station" three-dimensional non-contact profilometer.

Elemental analysis of the surface layer with a depth resolution of $0.5-1.0 \mathrm{~nm}$ was carried out by Auger electron spectroscopy on "Shkhuna-2" spectrometer.

\section{Results and Discussion}

\subsection{The Investigation of Titanium Layer}

The thickness of titanium coatings obtained in the arc mode varied in the range of $5-40 \mu \mathrm{m}$. Figure 1 shows scanning electron microscopy (SEM) images of the samples with Ti coating.

The image analysis shows that there are individual crystallites of titanium and craters of different sizes on the surface of Ti coatings. The shape of the craters indicates that they originate because of the detaching of large microparticles. During the collision with surface of samples such microparticles are in the solid state of aggregation and also they have a low speed. It determines their low adhesive strength. In our case this effect is a positive feature, because it increases the effective area of the

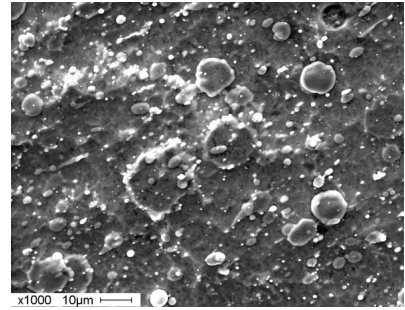

(a)

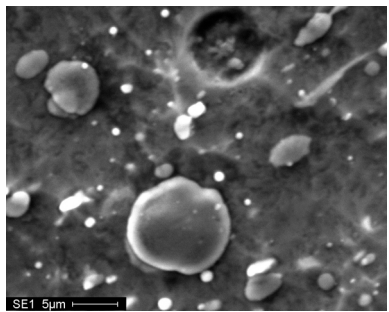

(b)
Figure 1. SEM of titanium coatings on stainless steel, magnitude: (a) $\times 1000$; (b) $\times 2500$. sample surface.

X-ray Diffraction (XRD) analysis of stainless steel basis shows the contents of $\mathrm{Fe}, \mathrm{Cr}$ and $\mathrm{Ni}$ compounds. Energy dispersive (EDS) analysis of the basis shows the presence of $\mathrm{Fe}-80.34 \%, \mathrm{Cr}-12.7 \%$, and $\mathrm{Ni}-6.97 \%$, it corresponds to the composition of the stainless steel 12X18H9T.

The chemical composition of the sputtered primer coating corresponds to the chemical composition of the cathode made of titanium VT1-0. The results of XRD analysis of the coating at different times of deposition $(15,30,60,120 \mathrm{~min})$ are shown in Figure 2.

Comparative analysis of the obtained radiographs shows that the coating with thickness of approximately $20 \mu \mathrm{m}$ has no peaks corresponding to elements of the basis material, so it can be stated that the primer titanium layer completely isolates the steel substrate.

According to Auger spectroscopy the basic elements of titanium coating in at. $\%$ are $\mathrm{Ti}-91.1 ; \mathrm{C}-2.2 ; \mathrm{N}-3.1$; $\mathrm{O}-3.6$. The total content of impurity elements in the coating does not exceed $10 \%$, which is a valid parameter during the implementation of forming the valve group

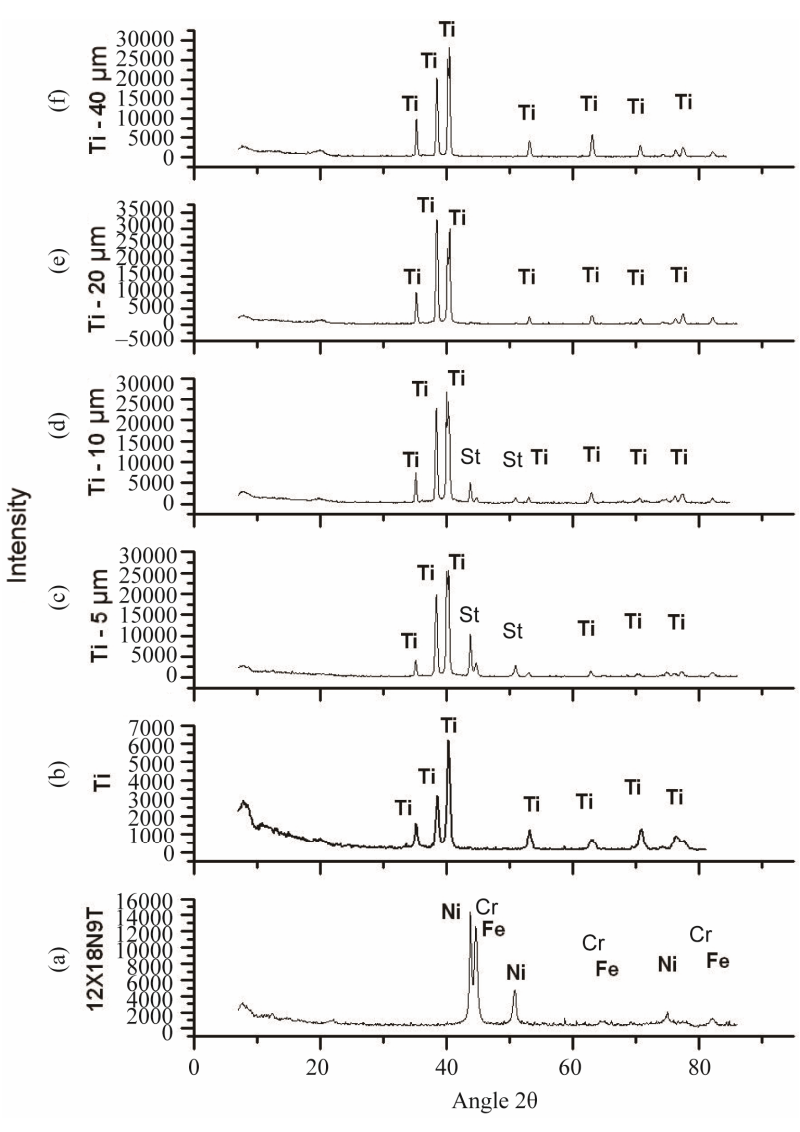

Figure 2. Radiographs: (a) The basis material (steel $12 \mathrm{X18}$ H9T); (b) Titanium VT 1-0; titanium coatings obtained at different times of deposition: (c) $15 \mathrm{~min}-5 \mu \mathrm{m}$; (d) $30 \mathrm{~min}-$ $10 \mu \mathrm{m}$; (d) $60 \mathrm{~min}-20 \mu \mathrm{m}$; (f) $120 \mathrm{~min}-40 \mu \mathrm{m}$; St-peaks corresponding to elements of the basis material. 
metal layer on the stainless steel surface.

The results of the study of steel substrate morphology and $\mathrm{Ti}$ coatings are shown in Table 1. Parameter Ra increases slightly with the increase of the coating's thickness.

The adhesive strength study shows that the destruction of the Ti coating with thickness $8.3 \mu \mathrm{m}$ begins at the critical load on the indenter of $11 \mathrm{~N}$. The observed pattern of the destruction indicates on slight internal residual stress levels, because the typical delamination along the grain boundaries near the track of the indenter is not observed.

\subsection{The Investigation of CaP Coating}

After the MAO process on steel substrates with the titanium primer layer, the thickness of formed $\mathrm{CaP}$ coating is about $7-15 \mathrm{~mm}$, porosity is $2 \%-8 \%$, and approximate pore size is $5-7 \mu \mathrm{m}$. The surface of $\mathrm{CaP}$ coating has a structure typical for the MAO process; SEM images are shown in Figure 3.

Conducted research shows that the $\mathrm{CaP}$ coatings formed by MAO method on the intermediate Ti layer do not reduce the strength properties of the basis material and the Ti primer. The microhardness of the amorphous $\mathrm{CaP}$ coatings is $3.11 \pm 0.82 \mathrm{GPa}\left(\mathrm{F}_{\max }=300 \mathrm{mN}\right)$, modulus of elasticity of the surface layer is $111 \pm 25$ $\mathrm{GPa}$. The roughness of the $\mathrm{CaP}$ layer (with the parameters $\mathrm{Ra}-0.88 \mu \mathrm{m}, \mathrm{Rz}-7.46 \mu \mathrm{m}$ ) is less than the roughness of the Ti primer.

The crystallization of the amorphous coating takes place in the temperature range $720^{\circ} \mathrm{C}-780^{\circ} \mathrm{C}$. The properties of the crystallized coating significantly change: roughness $(\mathrm{Ra}-2.38 \mu \mathrm{m}, \mathrm{Rz}-14.15 \mu \mathrm{m})$ increases, micro- hardness $\mathrm{H}=7.17 \pm 2.66 \mathrm{GPa}\left(\mathrm{F}_{\max }=300 \mathrm{mN}\right)$ and Young's modulus E $=60 \pm 43 \mathrm{GPa}$. The composition of the coating is formed with X-ray amorphous phases with a low content of crystalline nanostructured forms. On the surface of coatings there are spherulites with sizes up to $10 \mu \mathrm{m}$. The content of calcium integrally rises up to $23 \%$.

According to the results of XRD analysis (Figure 4) the chemical composition of the coatings after heat treatment changes, it is represented with various forms of titanium phosphates $\mathrm{CaTi}_{4}\left(\mathrm{PO}_{4}\right)_{6}, \mathrm{CaTiO}_{3}$ with the inclusion of calcium phosphates $\mathrm{CaP}_{2} \mathrm{O}_{6}$.

Table 1. The roughness parameters of the steel substrate and the Ti coating.

\begin{tabular}{cccc}
\hline The roughness & \multicolumn{3}{c}{ Studied surface } \\
\cline { 2 - 4 } $\begin{array}{c}\text { paramerer, } \\
\mu \mathrm{m}\end{array}$ & Steel substrate & $\begin{array}{c}\text { Ti coating, } \\
\text { thickness } 8.3 \mu \mathrm{m}\end{array}$ & $\begin{array}{c}\text { Ti coating, thickness } \\
23.3 \mu \mathrm{m}\end{array}$ \\
\hline $\mathrm{Ra}$ & 1.4 & 1.6 & 1.9 \\
$\mathrm{Rz}$ & 12.9 & 16.3 & 14.6 \\
\hline
\end{tabular}

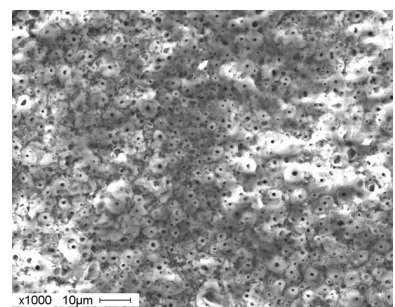

(a)

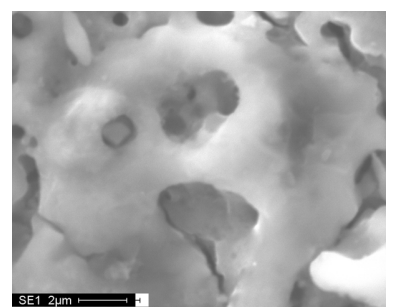

(b)
Figure 3. SEM of CaP coatings formed by MAO on the steel substrate with the titanium primer layer, magnitude: (a) $\times 1000$; (b) $\times 8000$.

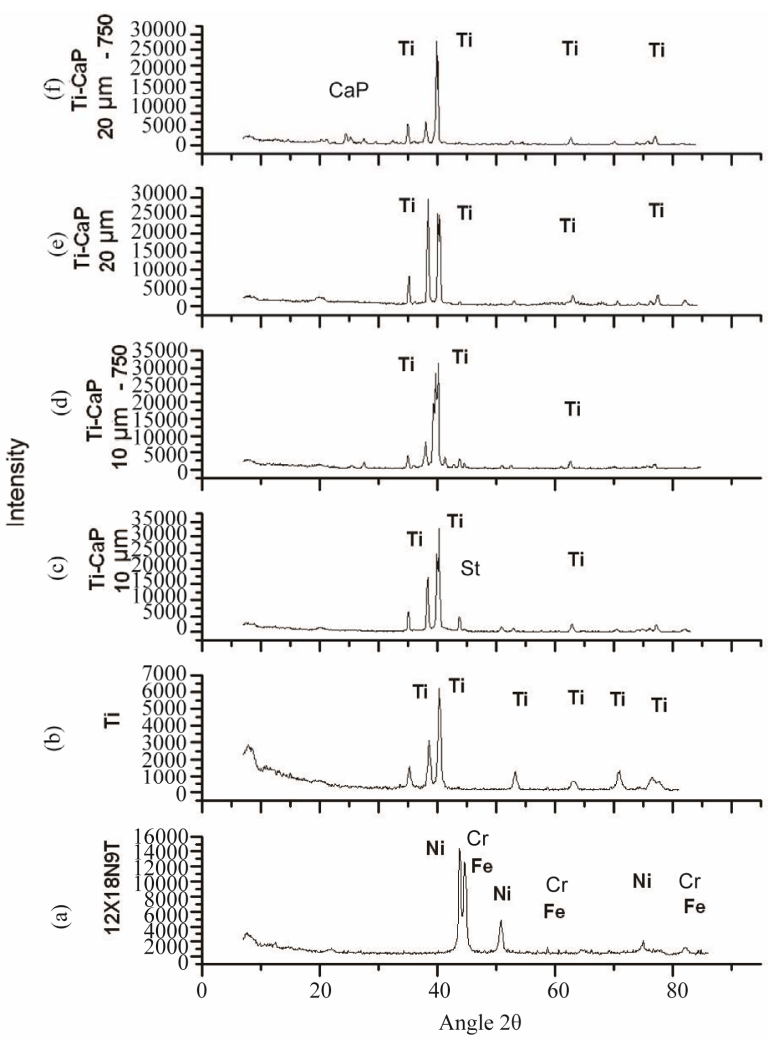

Figure 4. Radiographs: (a) The basis material (steel 12X18H9T); (b) Titanium VT1-0, MAO coating deposited on the titanium primer layer of thickness equal; (c) $10 \mu \mathrm{m}$, (d) 10 $\mu \mathrm{m}$ after the heat treatment at $750^{\circ} \mathrm{C}$; (d) $20 \mu \mathrm{m}$; (e) $20 \mu \mathrm{m}$ after the heat treatment at $750^{\circ} \mathrm{C}$; CaP-peaks corresponding to elements of the calcium-phosphate compounds.

According to energy dispersive analysis the elements on the surface of the biocomposite coating are $\mathrm{Ti}-47.88$ at. $\%, \mathrm{Ca}-9.16$ at. $\%, \mathrm{P}-12.39$ at. $\%$. The $\mathrm{Ca} / \mathrm{P}$ ratio is 0.74 . The low ratio of $\mathrm{Ca} / \mathrm{P}$ is connected, first of all, with the mechanism of the formation of MAO calciumphosphate coating. Such coating forms on Ti due to the process of oxidation and incorporation into its oxide of the electrolyte's components.

There are elements of the powders of hydroxyapatite and $\mathrm{CaO}$ dissolved in the electrolyte as well as the titanium primer layer on the Auger spectra of the biocom- 
posite (see Figure 5). The increased amount of carbon on the sample's surface can be explained by the contamination with technical oil during the mechanical sample preparation. The absence of chemical elements which compose steel provides the information that the substrate is completely isolated by multilayer biocomposite coating.

The results of energy dispersive analysis on different depth of the "steel substrate-biocomposite" system are summarized in Table 2.

The transition "steel-biocomposite" layer contains chemical elements of the substrate material (Fe, $\mathrm{Cr}, \mathrm{Ni}$ ) and titanium primer layer with a small amount of elements of $\mathrm{CaP}$ compounds. In the middle of the biocomposite $\mathrm{Fe}, \mathrm{Cr}$, Ni are absent, $\mathrm{Ti}$ is a key element, which proves the complete isolation of the substrate with the help of primer layer. The quantity of elements of $\mathrm{CaP}$ compounds increases closer to the surface of the biocomposite.

The transition "Ti primer layer-CaP coating" structure is shown in Figure 6. The titanium layer has a porous structure, $\mathrm{CaP}$ coating is denser than the Ti layer, and the border between them has no visible defects and exfoliations.

The ability of MAO calcium-phosphate coatings to stimulate the processes of osteoinduction is proven in the in vitro system using the PCR analysis to identify osteopontin, the specific bone marker and also in clinical practice. In the in vitro experiment The formation of the monolayer of mesenchymal stem cells (see Figure 7(a)) begins on the surface of $\mathrm{CaP}$ coating on the 14th day of the in vitro experiment, the emergence of immature bone tissue was observed after 30 days of the experiment (see Figure 7(b)).

Clinical studies show a positive consolidation of fractures, the absence of complications and relapses, bone regeneration, the absence of irritating, sensibilizing, general toxic and mutagenic action. The advantages of implants with MAO CaP coatings are especially marked during treatment of sedentary elderly patients [15].

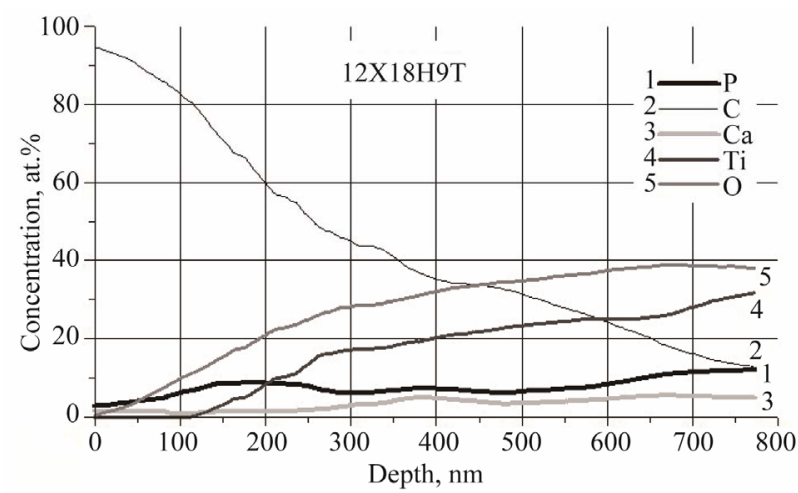

Figure 5. The distribution of elements on different thickness of the formed coating (auger spectroscopy).

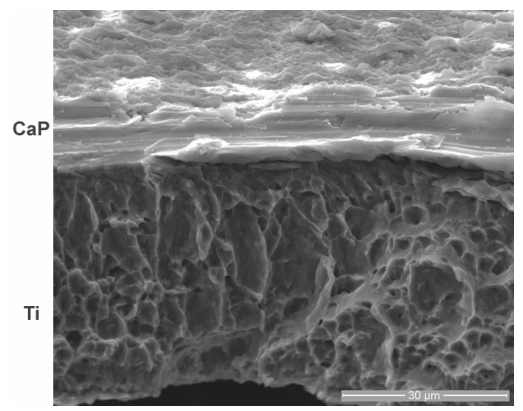

Figure 6. SEM of the biocomposite's transverse profile.

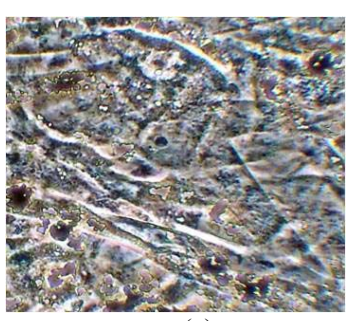

(a)

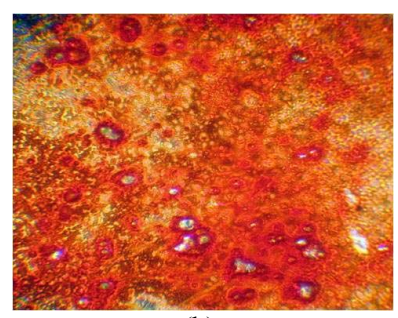

(b)
Figure 7. Histological sections of preparations obtained

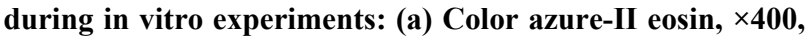
14 days; (b) The color of red alizorinovym, $\times 100,30$ days [15].

Table 2. The chemical composition of the "steel substratebiocomposite" system based on the results of energy dispersive analysis, at.\%.

\begin{tabular}{lccccccc}
\hline $\begin{array}{c}\text { The analyzed } \\
\text { region of the } \\
\text { system }\end{array}$ & $\mathrm{O}$ & $\mathrm{P}$ & $\mathrm{Ca}$ & $\mathrm{Ti}$ & $\mathrm{Fe}$ & $\mathrm{Cr}$ & $\mathrm{Ni}$ \\
\cline { 2 - 8 } & & & & & & \\
\hline $\begin{array}{c}\text { Biocomposite's } \\
\text { surface }\end{array}$ & 24.97 & 18.13 & 20.58 & 36.33 & - & - & - \\
$\begin{array}{c}\text { Half of the } \\
\text { biocomposite's } \\
\text { thickness }\end{array}$ & 6.17 & 11.23 & 10.18 & 72.42 & - & - & - \\
$\begin{array}{c}\text { The transition } \\
\text { "steel- } \\
\text { biocomposite" } \\
\text { layer }\end{array}$ & 1.22 & 0.91 & 0.51 & 2.46 & 70.27 & 5.88 & 18.8 \\
$\begin{array}{c}\text { The steel } \\
\text { substrate }\end{array}$ & - & - & - & - & 80.34 & 12.7 & 6.97 \\
\hline
\end{tabular}

\section{Conclusions}

The MAO method is used by a number of firms for the formation of $\mathrm{CaP}$ coatings on $\mathrm{Ti}$ implants, which have already demonstrated their effectiveness in clinical practice. In some cases, it is more preferable to use stainless steel and its alloys for large joint endoprostheses, because in comparison with $\mathrm{Ti}$ they have better strength properties, but lower biocompatibility.

The proposed hybrid method allows forming multilayer composite coatings which consist of titanium primer layer and MAO calcium-phosphate coating on stainless steel implants. 
The use of vacuum arc discharge allows the formation of the solid and homogeneous Ti layer on the surface of stainless steel and thickness of this layer is sufficient enough for the MAO process which is a key technique in the hybrid method.

The chemical composition of the biocomposite surface layer defined with the help of Auger, EDS and XRD spectroscopy is presented with compounds of calcium and phosphorus. The composite structure of the coating allows combining the properties of stainless steel good strength with high bioactivity of calcium-phosphate layer for bone regeneration.

It is expedient to use such biocomposites for implants which carry heavy loads and intended for long-term use, e.g. for total knee endoprostheses. The importance and significance of the hybrid method is substantiated by the cost-effectiveness and quality of the formed biocomposites.

\section{Acknowledgements}

The work is performed with the support of Federal Target Program (state contract No. 16.513.11.3075), RFBR (project No. 11-08-98032-r_sibir_a) and state order (project No. 7.1084.2011).

\section{REFERENCES}

[1] L. Galois and D. Mainard, "Bone Ingrowth into Two Porous Ceramics with Different Pore Sizes: An Experimental Study," Acta Orthopcedica Belgica, Vol. 70, No. 6, 2004, pp. 598-603.

[2] S. V. Dorozhkin, "Biocomposites and Hybrid Biomaterials Based on Calcium Orthophosphates," Biomatter, Vol. 1, No. 1, 2011, pp. 3-56. doi:10.4161/biom.1.1.16782

[3] J. C. Park, H. Ch. Lim, J. Y. Sohn, J. H. Yun, U. W. Jung, et al., "Bone Regeneration Capacity of Two Different Macroporous Biphasic Calcium Materials in Rabbit Calvarial Defect," Journal of Korean Academy of Periodontology, Vol. 39, 2009, pp. 223-230. doi:10.5051/jkape.2009.39.S.223

[4] C. Y. Ning, Y. J. Wang, W. W. Lu, Q. X. Qiu, R. W. Lam, et al., "Nano-Structural Bioactive Gradient Coating Fabricated by Computer Controlled Plasma-Spraying Technology," Journal of Materials Science: Materials in Medicine, Vol. 17, No. 10, 2006, pp. 875-884. doi:10.1007/s10856-006-0176-9
[5] Y. Bai, K. A. Kim, I. S. Park, S. J. Lee, T. S. Bae, et al., "In Situ Composite Coating of Titania-Hydroxyapatite on Titanium Substrate by Micro-Arc Oxidation Coupled with Electrophoretic Deposition Processing," Materials Science and Engineering: B, Vol. 176, No. 15, 2011, pp. 1213-1221. doi:10.1016/j.mseb.2011.06.019

[6] T. Troczynski and D. M. Liu, "Sol-Gel Calcium Phosphate Ceramic Coatings and Method of Making Same," US Patent No. 6426114, 2002.

[7] S. Sarangapani and P. D. Calvert, "Biomimetic Calcium Phosphate Ceramic Coatings and Method for Making the Same," US Patent No. 6129928, 2000.

[8] M. M. Dicu, M. Abrudeanu, J. P. Millet, S. Moga, V. Rizea, et al., "Physico-Chemical Properties of Microarc Oxidation of Biocompatible Coatings on Titanium: Influence of Electrochemistry Parameters," UPB Scientific Bulletin: B, Vol. 74, No. 1, 2012, pp. 193-202.

[9] L. H. Li, H. W. Kim, S. H. Lee, Y. M. Kong and H. E. Kim, "Biocompatibility of Titanium Implants Modified by Microarc Oxidation and Hydroxyapatite Coating," Journal of Biomedical Materials Research, Vol. 73, No. 1, 2005, pp. 48-54.

[10] S. H. Lee, H. W. Kim, E. J. Lee, L. H. Li and H. E. Kim, "Hydroxyapatite- $\mathrm{TiO}_{2}$ Hybrid Coating on Ti Implants," Journal of Biomaterials Applications, Vol. 20, No. 3, 2006, pp. 195-208. doi:10.1177/0885328206050518

[11] H. D. Steffens and M. Mack, "Plasma Spraying as an Advanced Tool in Surface Engineering," Pure \& Applied Chemistry, Vol. 62, No. 9, 1990, pp. 1801-1808. doi:10.1351/pac199062091801

[12] D. M. Brunette, P. Tengvall, M. Textor and P. Thomsen, "Titanium in Medicine: Material Science, Surface Science, Engineering, Biological responses and Medical Applications," Springer, New York, 2001.

[13] S. I. Tverdokhlebov, V. P. Ignatov, I. B. Stepanov, D. O. Sivin and V. P. Shakhov, "Calcium-Phosphate Bioactive Coating on the Implant and Method of Deposition," RF Patent No. 2423150, 2009.

[14] A. I. Ryabchikov and I. B. Stepanov, "Equipment and Methods for Hybrid Technologies of Ion Beam and Plasma Surface Materials Modification," Surface and Coating Technology, Vol. 203, No. 17-18, 2009, pp. 2784-2787. doi:10.1016/j.surfcoat.2009.02.126

[15] V. V. Aghajanian, S. I. Tverdokhlebov, E. N. Bol'basov, V. P. Ignatov and E. V. Shesterikov, "Osteoinductive Coatings Based on Calcium Phosphates and the Prospects for Their Use in the Treatment of Polytrauma," Polytrauma, No. 3, 2011, pp. 5-13. 